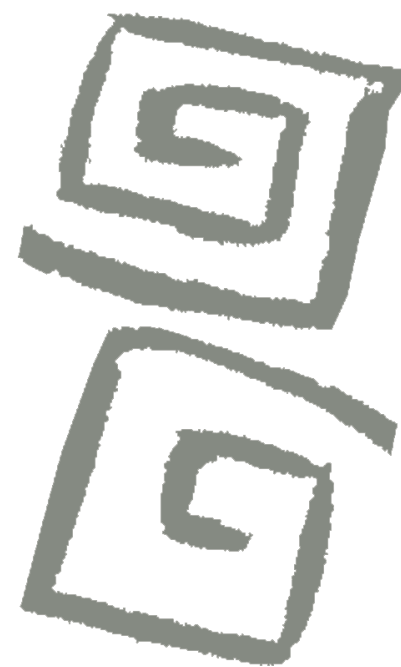

\title{
Varones adolescentes en un contexto rural mexicano: sufrimiento psíquico y cuidado entre pares
}

\author{
Adolescent males in a rural Mexican context: psychic \\ suffering and care among peers
}

Hernando Hernández-Nava', Guillermo Hernández González², Pamela Garbus $^{3}$

${ }^{1}$ Magíster en Educación para la Ciudadanía. Profesor de la Facultad de Psicología Universidad Autónoma de Querétaro. Santiago de Querétaro, México. $\square$ (iD

${ }^{2}$ Magíster en Psicología Social. Profesor de la Facultad de Psicología, Universidad Autónoma de Querétaro. Santiago de Querétaro, México. $\triangle$ (iD

${ }^{3}$ Autora de correspondencia: Doctora en Salud Mental Comunitaria. Profesora de Tiempo Completo, Facultad de Psicología, Universidad Autónoma de Querétaro. Miembro del Sistema Nacional de Investigadores. Santiago de Querétaro, México. $\bowtie$ iD
RESUMEN Se trata de un estudio exploratorio-descriptivo de corte cualitativo que se llevó a cabo en San Antonio de la Cal, comunidad de Tolimán, estado de Querétaro, México. Se buscó analizar las formas de sufrimiento psíquico y las prácticas de cuidado entre pares varones adolescentes de este contexto rural. Para ello se realizaron entrevistas semiestructuradas y un taller con varones adolescentes a partir de una muestra no probabilística y voluntaria. El estudio mostró que, para los participantes, las principales fuentes de sufrimiento psíquico son las relaciones con sus padres y sus novias. Tienen dificultades para discriminar y expresar emociones como la tristeza y el enojo. Las prácticas de cuidado entre pares son paradójicas en tanto son, al mismo tiempo, prácticas de riesgo. Todo esto se enmarca en el proceso de transformación de la dinámica de la ruralidad.

PALABRAS CLAVES Población Rural; Adolescente; Masculinidad; Salud Mental; México.

ABSTRACT An exploratory-descriptive study employing qualitative methods was carried out in San Antonio de la Cal, Tolimán, Querétaro, Mexico. The main purpose of the study was to analyze the forms of psychic suffering and care practices among adolescent male peers in a rural context. Nine semi-structured interviews were conducted, as well as a workshop with adolescent males selected from a non-probabilistic and voluntary sample. The study showed that the main sources of psychic suffering for participants included their relationships with parents and girlfriends. They presented difficulties in identifying and expressing emotions such as sadness and anger. Care practices among peers were paradoxical, as they simultaneously constituted risk practices. We interpret this in the context of transformations taking place in the dynamics of rurality.

KEY WORDS Rural Population; Adolescent; Masculinity; Mental Health; Mexico. 


\section{INTRODUCCIÓN}

Pensar la categoría salud y los fenómenos que se articulan en torno a ella, es una invitación a rebasar los paradigmas que explican los procesos de enfermedad como un problema individual y meramente biológico. Se trata de configurar una mirada que dé cuenta de las múltiples relaciones entre el sujeto y las condiciones sociales, económicas y culturales que derivan en procesos de bienestar integral y/o morbimortalidad colectivos. Para ello, se requiere generar conocimiento situado, que ponga énfasis en los elementos que matizan las lógicas de salud. Pensar la salud en contextos rurales representa la intersección de condiciones culturales de vida, de políticas públicas en diferentes niveles de organización y de experiencias de precariedad, inequidad y falta de atención institucional. Esto lleva a las personas que viven en estos contextos a generar estrategias colectivas de prevención y cuidado de la salud, más allá de los servicios proporcionados por los gobiernos, muchas veces inexistentes.

De manera particular, abordar la salud mental desde una dinámica colectiva impone retos de comprensión que rebasen la lógica psiquiátrica de "locura" intrapsíquica que deriva en prácticas de medicalización y hospitalización $^{(1)}$. Frente a esto, la Organización Mundial de la Salud (OMS) propone una visión socioeconómica, que vincula la salud a la capacidad productiva de las personas para "hacer una contribución a su comunidad"(2). Sin embargo, algunos autores cuestionan esta visión $^{(1,3,4,5)}$, en tanto consideran que invisibiliza el contexto social, cultural e histórico. La salud mental es un fenómeno complejo que requiere ser mirado desde la transdisciplina y no solo en términos de enfermedades o diagnósticos. Posicionarse en la definición de la enfermedad mental, limita la identificación de algunos padecimientos que no son necesariamente patologías mentales pero que producen sufrimiento ${ }^{(3)}$.

Este trabajo se sitúa en el marco de la salud colectiva toda vez que, en términos de Edmundo Granda, aceptamos el "esfuerzo por ver más allá del horizonte que nos ha legado la salud pública convencional"(6), y asumimos el desafío de "transformar nuestra acción en un quehacer humano profundamente, comprometido con la vida y con el cuidado de la enfermedad"(6). Este trabajo, retoma la idea de cuidado y se distancia del concepto de enfermedad, retomando en su lugar la categoría de sufrimiento psíquico ${ }^{(3)}$.

De lo anterior se deriva el interés en el sufrimiento psíquico como un elemento particular de la salud mental, que refiere a aquellas expresiones que no pueden ser encuadradas en la enfermedad objetivada; es decir, aquella información que manifiestan los sujetos sobre su malestar, que no encuentran lugar en la ciencia positivista y que es diferente en su forma individual y colectiva, por lo tanto, en la manera de padecerlo y nombrarlo, lo que permite la incorporación del nivel subjetivo del sufrimiento ${ }^{(3)}$.

Una de las etapas de desarrollo, en la que resulta importante explorar las formas de sufrimiento psíquico, es en la adolescencia, al ser una etapa crítica de transformación del rol de los sujetos en la comunidad. En torno a la adolescencia, entre los 13 y los 19 años, se suelen identificar una serie de posturas patologizantes e individualistas, ligadas a procesos del desarrollo psíquico en el binomio normalidad/anormalidad.

En esta etapa vital, la familia deja de ser el principal referente de socialización, y los sujetos se vuelcan al grupo de pares y hacia los espacios de socialización secundaria (la escuela, la calle y la comunidad) adquiriendo nuevas experiencias y significados acerca de su identidad personal pero también del submundo de las instituciones ${ }^{(7)}$.

Sin embargo, no se puede hablar de forma general de la experiencia adolescente, pues esta se encuentra diferenciada por múltiples condiciones, siendo una de ellas el género. La manera en que se recoloca una persona en el tejido de su comunidad dependerá (en un primer momento) de las consignas propias de ser hombre o mujer, generando diferencias en los roles sociales y las formas de socialización. De esta manera, en el caso de los varones, Hernández-Castañeda ${ }^{8}$ destaca 
que las relaciones están estrechamente vinculadas a los espacios de socialización, y lo explica desde el concepto de núcleos relacionales, haciendo mención a "las agrupaciones de sujetos con los que viven ligas afectivas, sentimentales, sociales y de intercambio"(8). Al decir de Vale de Almeida ${ }^{(9)}$, "la masculinidad hegemónica se constituye y reproduce a través de una serie de diferentes relaciones sociales y construcciones simbólicas" $y$, a diferencia de la feminidad que es vista como una esencia permanente vinculada a la maternidad, está en permanente construcción y confirmación.

A nivel mundial, la intersección entre masculinidad y salud ha cobrado relevancia en los últimos años pues, de acuerdo con Mitchell ${ }^{(10)}$, los hombres presentan mayores índices de morbilidad y mortalidad que se traduce en menor expectativa de vida. El 36\% de las muertes en varones pudieron evitarse en comparación con el $19 \%$ de las muertes en mujeres. Lo anterior se explica en buena medida debido a las prácticas diferenciadas en el consumo de sustancias como el alcohol y el tabaco, los riesgos laborales, la violencia y la subutilización de los servicios de salud.

Por su parte, la Secretaría de Salud de México ${ }^{(11)}$, identificó que los egresos hospitalarios entre hombres y mujeres de 10 a 14 años de edad no son muy distintos entre sí; sin embargo, en el grupo de edad de los 15 a 19 años aparece una notable diferencia, en tanto las mujeres tuvieron una mayor demanda de atención médica por motivos vinculados a la salud sexual y reproductiva, los hombres llegaron al servicio médico por traumatismos ocasionados en accidentes y violencias, envenenamientos, intoxicación por sustancias e intentos de suicidio; es decir, su morbimortalidad está asociada a determinantes sociales que tienen efectos en la salud y el bienestar. Estos factores, especialmente, entre los varones tienen una estrecha relación con el sufrimiento psíquico y otras esferas de la salud, como las prácticas de autocuidado y cuidado entre pares.

Frente a la relación entre sufrimiento psíquico y el resto de las lógicas de salud en los varones adolescentes, la noción de prácticas de cuidado se vuelve una posibilidad de análisis y de intervención. Para su conceptualización, se retoma a Figueroa y Flores quienes las definen como

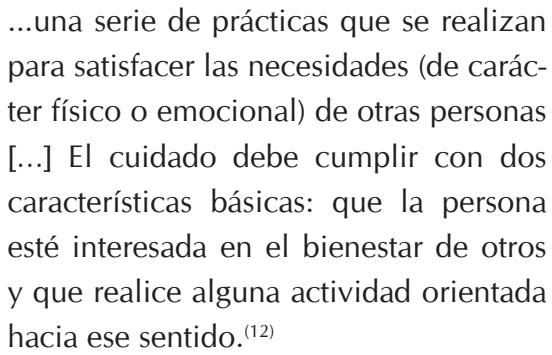

Entonces, las prácticas de cuidado en los varones no ocurren de manera automática o voluntaria, ya que no han sido socializados para ello, aunque suceden principalmente en situaciones de excepcionalidad y emergencia; es decir, remiten a casos en los que es fundamental actuar en términos de cuidado (el cuidado de los padres en situaciones extremas como la discapacidad o enfermedad, etc.) y que fracturan el modelo tradicional de ser varón, en el que se pueden construir nuevos significados, valores y una ética del cuidado en las relaciones y las prácticas ${ }^{(12)}$. De esta manera, al explorar las prácticas de cuidado, se busca destacar la importancia que tienen en un proyecto de salud mental colectivo que enfatice el lazo entre pares de varones adolescentes.

Como se ha planteado, la propuesta de salud mental colectiva requiere pensar el conocimiento situado, que tome en cuenta las condiciones de vida, en este caso, de la comunidad rural San Antonio de la Cal, del estado de Querétaro, México. El interés por la ruralidad está fundamentado en las condiciones de inequidad en el acceso a los servicios de salud, así como en las condiciones económicas, laborales y de calidad de vida propias de la ruralidad y de este municipio particularmente. Según Jarquín et al. ${ }^{(13)}$, poner la mirada en la ruralidad contemporánea exige dar cuenta de los procesos de desagrarización, en los que las familias dejan de centrar su economía en la producción agrícola o ganadera local para formar parte de las lógicas de producción del sector secundario 
o terciario, lo que por una parte genera cambios en la organización familiar y el tejido de la comunidad y, por otra, aumenta las lógicas de desigualdad, pues el acceso a servicios o instituciones públicas sigue bajo patrones de aislamiento $^{(14)}$ mientras que el empleo que se puede conseguir se puede caracterizar como precario. Lo anterior se imbrica en nuevas formas de sufrimiento psíquico, tal como se presentará en este trabajo.

La localidad de San Antonio de la Cal es una de las tres delegaciones del municipio de Tolimán, municipio con bajas condiciones de desarrollo económico y social, que se encuentra en la zona centro-oeste del estado de Querétaro, México, aproximadamente a $65,5 \mathrm{~km}$ de distancia de la capital del Estado. El poblado se encuentra a un costado de la Peña de Bernal, elevación rocosa que atrae al turismo nacional e internacional del Estado y forma parte de municipio de Ezequiel Montes. Sin embargo, San Antonio no goza de popularidad turística y, por lo tanto, tampoco de las condiciones económicas y de desarroIlo social que el turismo genera. De acuerdo con el Consejo Nacional de Evaluación de la Política Social (CONEVAL), la comunidad está catalogada con alta marginación ${ }^{(15)}$, gran parte de la población adulta y joven de esta comunidad se emplea en puestos de baja paga en los negocios turísticos del pueblo vecino, generando riqueza para otro municipio y perpetuando las condiciones de inequidad.

Según el Censo de Población y Vivienda del Instituto Nacional de Estadística y Geografía (INEGI) ${ }^{(16)}$, en el Municipio de Tolimán se registraron 26.372 habitantes, de los cuales 2.721 radican en la localidad de San Antonio de la Cal, y representan el $10,3 \%$ de la población total del municipio. La encuesta intercensal del INEGI ${ }^{(16)}$ reportó que, de esos 2.721 habitantes, 1.388 son mujeres $(51 \%)$ y 1.333 varones (49\%). El estudio se enfocó en la población masculina entre los 15 y 19 años de edad, que representan el 7,9\% de la población total.

Es importante destacar que la comunidad forma parte de la zona con mayor población indígena del Estado. Asimismo, el INEGI reportó que en Tolimán hay 5.752 personas de cinco años y más que hablan alguna lengua indígena ${ }^{(16)}$, y destacan principalmente el hñähñú. No obstante, para los participantes, la generación de sus abuelas es la última tradicional indígena; y refieren que ellos no se reconocen como indígenas, ni conocen palabras de la lengua de sus abuelas.

Este trabajo se enmarca en una investigación titulada Prácticas de cuidado de la salud mental entre pares como un componente necesario para la construcción de ciudadanía activa de los hombres adolescentes: Un estudio exploratorio-descriptivo ${ }^{(17)}$, que se llevó a cabo como parte de los requisitos para obtener el título de Maestría de uno de los autores, el cual fue dirigido por la coautora y asesorado por otro coautor. Anteriormente se han escrito dos trabajos que se pueden considerar fragmentos de la tesis: un capítulo de libro, titulado Prácticas de cuidado de la salud mental entre pares: componente necesario para la construcción de ciudadanía activa de los hombres adolescentes ${ }^{(18)}$, cuyo objetivo fue conocer la forma en la que las prácticas de cuidado entre pares pueden favorecer el ejercicio de una ciudadanía activa en construcción, y se utilizó una metodología mixta. Por otro lado, se presentó un trabajo en el IX Congreso Internacional de Investigación y Práctica Profesional en Psicología XXIV Jornadas de Investigación de la Facultad de Psicología XIII Encuentro de Investigadores en Psicología del MERCOSUR, titulado Prácticas de cuidado de la salud mental entre pares en hombres adolescentes de San Antonio de la Cal, Qro.Mex ${ }^{(19)}$, cuyo objetivo fue analizar los datos arrojados por un cuestionario cerrado que se aplicó a la población de estudio con el objetivo de tener una primera aproximación a las características sociodemográficas de la población.

Este trabajo, puntualmente, busca problematizar el sufrimiento psíquico desde una visión compleja que dé cuenta de los elementos estructurales que intervienen en la salud mental y generan problemáticas en los sujetos concretos, así como pensar en el potencial del cuidado entre pares como acciones locales y situadas que disminuyan el impacto de la desigualdad social. Puntualmente, el 
objetivo de este artículo fue analizar las prácticas de cuidado entre pares y el sufrimiento psíquico en varones adolescentes de un contexto rural.

\section{METODOLOGÍA}

Se realizó un estudio exploratorio-descriptivo de corte cualitativo, que buscó profundizar en la visión subjetiva del malestar de los varones adolescentes, así como explorar los recursos o intentos de solución de la población estudiada. Se trabajó con los varones inscritos en el Colegio de Bachilleres ubicado en San Antonio de la Cal, que cuenta con una población total de 251 estudiantes, de los cuales 104 son varones.

Para recabar la información se utilizó un muestreo no probabilístico y voluntario. Se recurrió a dos técnicas de construcción de datos. La primera de ellas, que se inscribe dentro de las metodologías narrativas, fue una entrevista semiestructurada. Entre los meses de febrero y junio de 2017 se llevaron a cabo 9 entrevistas, las cuales se gestionaron a través de una invitación abierta que los investigadores realizaron en el salón de clases, enfatizando la participación voluntaria.

La segunda técnica, derivada de las metodologías participativas, fue un taller diagnóstico llevado a cabo en una sesión de 8 horas de duración, el 28 de junio de 2017, el cual buscaba problematizar y encontrar resonancia de los elementos encontrados en el primer instrumento a partir de la interacción de diferentes varones. En el taller se buscó que, desde la interlocución, se construyeran de forma colectiva los sentires, pensamientos, así como las necesidades sentidas y los intentos de solución de estas necesidades de los participantes.

Para la convocatoria y selección de los participantes del taller, se retomó la lista de jóvenes estudiantes que habían manifestado interés en seguir colaborando con la investigación. El taller contó con la asistencia de siete varones, entre los 16 y 18 años. A partir de una serie de técnicas grupales se exploraron las necesidades sentidas en torno al sufrimiento psíquico y la identificación de sus fuentes, la idea sobre los riesgos para la salud de hombres y mujeres, así como las prácticas de cuidado de sí y del otro desde su experiencia de vida.

La sistematización de los datos se realizó a partir de las transcripciones de las entrevistas y la relatoría del taller diagnóstico, y posteriormente se construyeron categorías analíticas por medio del uso del software ATLAS.ti 8.3.1.

Para el desarrollo de este estudio se respetaron los cuatro principios fundamentales en ética de la investigación científica: autonomía, justicia, no maleficencia, beneficencia. Se solicitaron los consentimientos informados a los padres de los participantes menores de edad, y el asentimiento a los jóvenes. Asimismo, se contó con autorización institucional para ingresar a la escuela a solicitar participación de los jóvenes.

\section{RESULTADOS}

Se presentan dos grandes categorías analíticas que se obtuvieron a partir del análisis de las técnicas aplicadas: las fuentes de sufrimiento psíquico y las formas de cuidado entre pares, las cuales son parte del objetivo de este trabajo.

Los participantes reconocieron que el sufrimiento psíquico es expresado con frecuencia a través del llanto, el consumo del alcohol o, incluso, a través de la violencia.

Respecto a las fuentes de sufrimiento psíquico, los participantes refirieron sentimiento de abandono y distancia emocional ante la salida de madres y padres al campo laboral sobre todo, y muy especialmente, con relación a la madre. Cabe mencionar que las madres de los participantes son parte de las primeras generaciones que se ven obligadas a salir de la comunidad a trabajar de manera remunerada. Los adolescentes varones reclaman y viven sensaciones de "abandono" y "distancia emocional" por esta situación, que vivencian como una falta de cumplimiento de los roles tradicionales asignados a las mujeres 
en la comunidad (crianza, trabajo doméstico, cuidado de los hijos/as). De hecho, la salida laboral materna no podría ubicarse más que como obligatoria para ellas, como consecuencia de las condiciones económicas, en la mayoría de los casos. En el relato de los jóvenes, el espacio de la mujer sigue siendo el del hogar y, el del hombre, de proveedor económico.

La figura paterna en sí es para muchos de ellos una fuente de sufrimiento también, en tanto lo perciben como distante, ausente o amenazante. La relación que tienen con el padre es, en general, compleja. Identifican esa distancia emocional y la atribuyen específicamente al trabajo y sus efectos en la vida cotidiana: agotamiento físico, desgaste emocional y aparente falta de interés en la vida afectiva.

No obstante, varios adolescentes reconocieron que sus padres se enfrentan a largas jornadas laborales en el sector terciario, en la zona turística cercana, o en las pocas fábricas que hay alrededor de la comunidad. Estas actividades son relativamente nuevas en la vida comunitaria que anteriormente se dedicaba al trabajo en la calera (mina de cal) que estaba dentro de la comunidad. De esta forma se observa cómo los cambios en la dinámica rural se entretejen en las nuevas configuraciones familiares, las cuales provocan en los adolescentes sentimientos de ausencia, distancia y de falta de apoyo familiar.

Estos cambios son un factor más que se suma al contexto de precariedad en el que están inmersos los adolescentes, que se sostiene en la falta de recursos económicos y de fuentes de empleo en la misma comunidad y municipio. Es preciso mencionar que el trabajo turístico que genera empleo para los habitantes de este municipio, se desarrolla en el municipio vecino por lo que los habitantes de San Antonio de la Cal son mano de obra de dicha actividad, siendo que su municipio no recibe más ganancias que las de sus propios salarios, los cuales no están muy por encima del salario mínimo y son, en la mayoría de los casos, provenientes de propinas y/o comisiones.

Así, el precario ingreso familiar, obliga a los jóvenes a transformar su rol social y salir tempranamente al ámbito laboral para aportar ingresos económicos a su familia de origen, o bien, para sacar adelante las propias necesidades, que es el caso de un informante de 19 años que es padre y sostiene el hogar económicamente. Esto se contradice con la expectativa histórica de los jóvenes en contextos urbanos de ser estudiantes y dedicarse a procesos de socialización típicos de su edad: salir con amigos, novias, etc. Frente a las nuevas lógicas laborales a las que se enfrentan, es preciso enfatizar que se trata de una comunidad rural con pocas posibilidades e intenciones de migración, la mayoría de los adolescentes aspiran a quedarse en la comunidad.

Las relaciones de pareja o noviazgo son otra de las principales situaciones que los adolescentes varones identificaron como productoras de sufrimiento psíquico. Por un lado, manifestaron sentir la exigencia de sus pares varones de vincularse sexual o afectivamente con más de una pareja, lo que representa el mandato cultural del contexto. Sin embargo, esta situación les produce confusión, rechazo y/o culpa ya que, a la par, y como ejemplo de otro de los cambios que se viven en la ruralidad, los discursos sobre el noviazgo que les presentan las instituciones públicas (de educación, salud, juventud, etc.) se contraponen con dicho mandato.

En torno al noviazgo, también se identificó una tensión entre el ideal de las relaciones bajo los roles de género tradicionales en los que fueron socializados y la manera en que, desde su percepción, las mujeres jóvenes se comportan en la actualidad. Por ejemplo, refirieron que las mismas consumen tanto alcohol como ellos -aunque no lo hacen en el espacio público como los varonesdicen groserías, tienen relaciones sexuales "a temprana edad" y toman la iniciativa invitándoles a beber a los varones. Esta transformación en la percepción acerca del rol de las mujeres produce en ellos confusión sobre la manera de vincularse amorosamente.

Respecto a la expresión de sentimientos, a través del dispositivo grupal, los participantes pudieron dar cuenta de su incapacidad para identificar sus propias emociones, confundiendo y expresando de manera indistinta el enojo y la tristeza. 
Respecto al otro tema central de este trabajo, que se centra en indagar acerca de sus prácticas de cuidado, entendidas como todos aquellos testimonios que incorporen actividades o acciones destinadas a atender necesidades físicas o emocionales de otros ${ }^{(11)}$-en este caso, otro varón de su grupo de pares- se observó en ellos una necesidad y deseo de expresar su malestar o sufrimiento psíquico. Esto los coloca en una situación de permanente contradicción, pues resaltaron el vínculo de amistad que tienen con sus pares varones, el cual se traduce en apoyo, palabras positivas o consejos pero, al mismo tiempo, expresaron dificultades para establecer vínculos más íntimos y cercanos entre ellos, que les permitan conversar sobre situaciones más personales, concretamente vinculadas a su malestar ${ }^{(18)}$.

Una de las consecuencias de esta contradicción se muestra en la búsqueda de cuidado y apoyo en sus pares mujeres, pues perciben una incapacidad en ellos mismos y en sus pares, en tanto varones, de ser comprensivos al tiempo que depositan en las mujeres características como la empatía, la escucha e interés en el otro. Evidentemente, les resulta más fácil establecer la confianza necesaria para abrirse y contar sus asuntos personales con las mujeres que con sus pares varones pues, el vivir en una comunidad pequeña, con características como la de este estudio, la burla o exhibición indolente por parte de los varones se vuelve un peligro constante, situación que se expresa en la frase popular del contexto rural "pueblo chico, infierno grande".

Frecuentemente, frente al malestar propio o de algún amigo, los participantes refirieron recurrir al consumo de alcohol para sentirse autorizados a mostrarse vulnerables o simplemente para incentivar ese encuentro entre pares: "si está triste le invito una chela [cerveza]". Es el mandato de género que determina que el alcohol "como que les hace sentirse más a los hombres" o que "desinhibe para hablar de lo íntimo".

Este conjunto de creencias, interiorizadas a partir de la socialización de género, están presentes en los hombres adolescentes y, paradójicamente, son determinantes para intentar alcanzar el bienestar, por lo que el consumo del alcohol como catalizador del sufrimiento psíquico, limita alternativas para sobrellevar los embates de la vida cotidiana.

Cuando se les preguntó a los adolescentes varones por el significado que tienen del concepto de cuidado, estos lo asociaron de manera inmediata con protección, "no andar o dejar sólo a un amigo", especialmente en situaciones de riesgo o de pelea con otros. Este concepto se rastrea como una consigna de la masculinidad tradicional; sin embargo, de una manera situada, el cuidado como compañía ante la violencia se matiza por las lógicas de relación entre varones propias de su contexto, marcada por rivalidades entre diversas comunidades, o por pugnas entre barrios de la misma comunidad.

En general los participantes manifestaron que los bailes o fiestas de su comunidad terminan en peleas, desarmados o con armas blancas. Sin embargo, los participantes refirieron sentirse incómodos peleando y manifestaron que desearían evitar pelear, situación que les resulta imposible si algún otro agrede a su par varón: "si lo están golpeando ni modo de no meterme".

Concretamente, en relación con las prácticas de cuidado, se encontraron aquellas vinculadas al consumo de alcohol como mediador de la convivencia, la protección del otro a través de la violencia como medio para resolver conflictos, la utilización del espacio de la calle como espacio de socialización secundaria del grupo de pares, donde suelen darse las peleas con otros considerados rivales. Como puede observarse, todas estas prácticas se ubican más en las coordenadas de las prácticas de riesgo que de cuidado. Sin embargo, paradójicamente, ellos mismos leen sus prácticas de cuidado entre pares enlazadas a estas mismas acciones.

En resumen, tanto en la protección como en el consumo de alcohol, las prácticas de cuidado entre pares en estos varones son, al mismo tiempo, prácticas de riesgo que Ilevan a estos adolescentes a experimentar sentimientos paradójicos frente al cuidado de pares. 


\section{DISCUSIÓN}

Un estudio atravesado por una perspectiva de género en un contexto específico debe considerar diferentes variables como la edad, la etapa del curso vital y la condición económica del contexto $^{(20)}$. Si bien los varones adolescentes no son todos originarios de San Antonio de la Cal, pues vienen de comunidades del municipio de Tolimán, todas estas son también áreas rurales con una presente tradición indígena. Sin embargo, es preciso plantear que ninguno de los varones que participaron de este estudio se identifica como parte de la población indígena, de hecho, ninguno habla hñähñú.

Para Núñez Noriega(21), las relaciones entre pares son claramente pujas de poder determinadas por las consignas de género y validadas simbólicamente por el Estado. Pensando a este como una institución patriarcal que "feminiza", minimiza y desprecia lo indígena, es preciso mencionar que los participantes estudian el nivel medio superior en una escuela pública, no-multicultural -al igual que todas las que existen en el país en este nivel educativo- que los forma en contenidos con una impronta globalizadora en la que incorporan, por ejemplo, como segunda lengua, el inglés que les hace mirarse desde el desarrollo frente a la ruralidad y su origen indígena. Considerando a la escuela como institución subjetivante ${ }^{(22)}$, incorporan la lógica del desarrollo y progreso que es contraria a lo rural y lo indígena.

La familia tradicional biparental con hijos es la principal configuración familiar en la región, aunque también encontramos un porcentaje significativo de jóvenes que viven únicamente con la madre, jefa de familia ${ }^{(18)}$. En ambos casos, muchos jóvenes reportaron que sus madres tienen alguna actividad económica que les permite participar en el ingreso familiar y que muchos de estos trabajos son precarios (venta de golosinas, gelatinas o alimentos). Esta situación pone en conflicto a los adolescentes en tanto han crecido con el estereotipo de género que iguala masculinidad a proveeduría única del hogar y se tensa con la incapacidad de cubrir esta consigna en sus padres y probablemente en ellos.

Un aporte de Burin y Meler ${ }^{(23)}$, respecto a la identidad subjetiva de los varones y respecto al malestar que con frecuencia presentan, está relacionado precisamente con este encargo cultural de género acerca de la proveeduría del hogar. Dicho mandato se enfrenta a un contexto global en América Latina y, de manera más palpable en esa región de Tolimán, Querétaro, donde el trabajo como actividad económica recibe ingresos cada vez más precarios y donde existe un incremento de las tasas de desempleo, en un contexto de crisis económica.

Un dato relevante respecto a la actividad económica que realizan los jóvenes es que esta ocurre en el pueblo de Bernal que, como hemos dicho, se trata de una localidad que cuenta con el monolito más grande de México, lo que la convierte en un atractivo destino turístico y, por lo tanto, genera fuentes de empleo para los jóvenes de la región. Los trabajos que realizan principalmente son en restaurantes, como meseros y en los servicios turísticos en general. Debido a las condiciones del contexto que ya fueron mencionadas, el ingreso que ellos perciben en sus trabajos permite aligerar el peso del gasto de sus familias.

Retomando lo mencionado anteriormente sobre el contraste generacional que existe entre estos jóvenes y sus padres y abuelos, específicamente con relación al empleo y la identidad indígena, es posible leer esta realidad como efecto de la interacción con otras figuras de diferentes espacios sociales (trabajo, escuela), que no pertenecen a sus generaciones anteriores ${ }^{(24)}$.

Aunque la proveeduría sigue siendo parte fundamental de la configuración masculina en San Antonio de la Cal, las formas y condiciones del ejercicio de esta se están transformando en relación con las exigencias laborales ${ }^{(25)}$.

El contraste que encontramos es el cambio de actividad entre los padres y los hijos pues, mientras los primeros han aprendido oficios que conllevan el uso del cuerpo y la fuerza (albañilería, obreros de la calera, herreros, carpinteros), los hijos realizan de actividades en las que el cuerpo no está expuesto 
necesariamente al mismo desgaste y riesgos. Además, si bien dichas actividades exigen otro tipo de habilidades como las de atención al cliente, higiene y aspecto personal, carisma e incluso el manejo de alguna computadora y del idioma inglés, enfrentan otro tipo de desgastes como el estrés de tratar con "clientes difíciles y demandantes" o con horarios de trabajo extenuantes.

Estas actividades económicas y condiciones del contexto modifican la vida rural de la comunidad en la que viven y con ello la vida social. Asimismo, la construcción de género de los hombres adolescentes de la región parece estar tornándose más flexible pero también más contradictoria: aún cuando persisten ideas y creencias tradicionalistas vinculadas a lo que debe ser o hacer un hombre o una mujer, surgen -aunque aún de manera incipiente- planteamientos sobre los derechos y la libertad de las mujeres al trabajo, incluso a la necesidad de que los hombres se coloquen de una manera diferente frente a este nuevo escenario social.

En buena medida, la socialización de género para ellos, así como las condiciones económicas y la edad, constituyen un elemento que podemos pensar desde la categoría de interseccionalidad sugerida por Mara Viveros $^{(26)}$. La masculinidad desde la perspectiva interseccional reconoce que

\footnotetext{
...la clase social, la pertenencia étnica, el ciclo de vida o la orientación sexual son factores de diferenciación de las identidades genéricas, entendemos la masculinidad como una realidad múltiple en función de los momentos históricos y de estos distintos elementos. ${ }^{(24)}$
}

Considerando el conocimiento como una práctica situada, se busca apelar a la mencionada interseccionalidad para evitar pensar de manera estática categorías tales como clase, origen, pertenencia étnica o edad. Es preciso dar cuenta del movimiento de estas categorías en los sujetos concretos y sus contextos para el estudio del objeto salud/salud mental.

Desde el inicio, este trabajo se centró en la comprensión de la importancia que tienen los grupos de pares para los varones, principalmente, durante la adolescencia. Al respecto recuperamos el componente de la amistad que se manifestó con frecuencia durante las entrevistas. La amistad juega un rol fundamental en las experiencias de la adolescencia, específicamente en el grupo de pares. Orcasita y Uribe ${ }^{(27)}$ y Rodríguez et al. ${ }^{(28)}$ establecen la importancia que tiene la afectividad y la confianza para el bienestar de los adolescentes. La amistad tiene además una función para el desarrollo de una ética del cuidado entre pares, por lo tanto, tiene un carácter político y transformador. Sin embargo, para los entrevistados, existe un límite en la confianza que solo pueden sobrepasar a partir de la ingesta compartida de alcohol. Para el estudio de la amistad entre varones, Gilmore -citado por Vale de Almeida $^{(9)}$ - propone la diferenciación entre amistad, compromiso y amistad de confianza. La amistad resulta de la interacción en un espacio que se considera neutral, que culmina con el "salir de farra". El compromiso va desde el bar hacia el hogar, generando expectativas de apoyo mutuo en caso de que sea necesario y, la amistad de confianza conllevaría una confidencia mutua de sus secretos personales, debiendo basarse en el afecto y en el dejar de lado los intereses personales poniendo en foco los del amigo. Así, Gilmore, distingue los espacios en los que se da la interacción y el nivel de compromiso e intimidad ante ese vínculo. Al respecto, se pudo observar que para estos jóvenes una cosa es ser compañeros de clase y juntarse para hacer tareas de la escuela y otra muy distinta compartir unas cervezas en el arroyo con las novias, por ejemplo, siendo esta una actividad que desarrollan con varones que consideran más cercanos.

Vela de Almeida ${ }^{(9)}$ retoma a Papataxiarchis, quien plantea que el fenómeno de la amistad masculina no puede ser solamente visto en términos económicos o políticos. El autor, en lugar de ver esta amistad como el apéndice de la estructura androcéntrica, la considera como un aspecto de la antiestructura, conectada con el ocio, el compañerismo -tanto en el consumo del alcohol como en las apuestas- y está caracterizada por la ausencia de funciones económicas. 
De hecho, en los jóvenes de San Antonio de la Cal, la amistad tampoco se establece sobre un aspecto económico de intercambio, pese a que es fundamental en la construcción de la subjetividad masculina, dado el momento del curso de sus vidas y en el espacio rural en el que viven. Ellos distinguen entre los compañeros de la escuela, a quienes a veces les dicen amigos, pero con los cuales no tienen la suficiente confianza para expresarse emocionalmente; esa expresión es efectivamente exclusiva ante algunos vínculos, en algunos momentos específicos y ante ciertas circunstancias. Así, el dispositivo escolar les permite conocer a más personas, pero no necesariamente establecer con ellos mayor intimidad o cercanía, esa relación estará efectivamente mediada por espacios abiertos como el arroyo, la calle o la casa de algún "compa", pero siempre con la presencia de alguna bebida alcohólica.

De hecho, el alcohol en estos hombres adolescentes juega un papel específico para la expresión afectiva entre pares, además para la expresión del sufrimiento psíquico. Así como para los hombres estudiados por Vela de Almeida en una zona rural de Portugal a finales de la década de 1980, tomar era una actividad que debía hacerse con otros varones y compartir y pagarles rondas de alcohol a otros era una práctica de ejercicio y construcción de una masculinidad hegemónica, en nuestro estudio, se encontraron posturas que revelan que, si bien los pares vinculados por la amistad pueden establecer prácticas de bienestar asociadas al cuidado, a estos varones estas prácticas los llevan a desarrollar conductas disruptivas y prácticas de riesgo, asociadas especialmente al hecho de que el afecto en estas relaciones solo puede manifestarse a partir de las conductas masculinas tradicionales ${ }^{(29)}$, como la ingesta excesiva de alcohol. Siguiendo a Gilmore podríamos decir que, si bien mencionan elementos que son afines a una amistad de confianza, compartir el sufrimiento psíquico con pares varones se da también a través del alcohol, el cual es facilitador de la expresión emocional por parte de los amigos pares. La expresión de las emociones puede darse con el alcohol como mediador pero, también, la incomodidad que puede ocasionar la escucha del sufrimiento del amigo es aminorada por la bebida.

Así, las lógicas de confianza entre varones parecen estar limitadas por la masculinidad hegemónica tanto en su expresión como en su escucha y es el alcohol la única vía dentro del espectro de masculinidad.

Gran parte de las expresiones vertidas sobre sus emociones y afectos fueron obtenidas a partir de la técnica de taller empleada, y no en las entrevistas cara a cara con un entrevistador varón. Esto hace suponer que el dispositivo grupal brindó posibilidades que no tienen en la vida cotidiana, aportando elementos para considerar que este tipo de experiencias pueden derivar en intervenciones que posibiliten reflexionar las masculinidades con hombres adolescentes desde una perspectiva interseccional.

Un asunto que se pudo constatar es la dificultad que presentan los hombres adolescentes para establecer precisamente estos vínculos afectivos y de intimidad entre pares, situación que se confirma con varios estudios en distintas latitudes del mundo, respecto a las relaciones intragénero ${ }^{(9,24,30,31,32)}$. Lo anterior está estrechamente vinculado con lo que estos autores Ilaman homo-sociabilidad o cultura de pares cuya creencia mitológica de base se encuentra en el machismo homofóbico $^{(33)}$ que supone que los hombres solo pueden establecer relaciones de complicidad, camaradería y de crueldad, pero difícilmente de cuidado, ternura y afectividad, lo que se presenta como un factor de riesgo para el cuidado de su sufirimiento ${ }^{(29,30)}$.

Niobe Way ${ }^{(34)}$ menciona que hay una intimidad entre los adolescentes varones durante la adolescencia y que los niños comparten sus secretos y sentimientos más profundos con sus amigos varones más cercanos. Sin embargo, a medida que los niños se convierten en hombres pierden estas amistades, lo que los lleva a sentirse aislados y solos. Es que al final de la adolescencia, refiere la autora, los niños sienten que deben volverse independientes y pasan a considerar que las emociones vulnerables y las amistades íntimas son exclusivas 
de las niñas y de los varones homosexuales. En los jóvenes de este estudio parece darse este fenómeno cuando refieren que es más fácil compartir sus sentimientos con las mujeres porque consideran que ellas están dotadas de condiciones, casi naturales, para escuchar, comprender y cuidar. Los varones pueden hacerlo, tal como se mencionó, solo a través de la confianza que habilita el alcohol entre vínculos muy cercanos.

Respecto de las causas de sufrimiento psíquico, resulta llamativo que las relaciones de pareja o noviazgo sean una de las principales situaciones que los adolescentes varones identificaron como productoras de sufrimiento psíquico. Sin embargo, dicho sufrimiento está mediado por los mandatos de una masculinidad hegemónica que les demanda vincularse sexual o afectivamente con más de una pareja, demandas que se contraponen con las nuevas exigencias sociales que son efecto de los cambios que se viven en la ruralidad, a partir de la llegada de nuevos discursos sobre el noviazgo por medio de las instituciones públicas, principalmente, la escuela. Así, el ejercicio de la sexualidad e involucrarse en relaciones amorosas representa para ellos el problema de la contraposición de diferentes mandatos a los que creen tener que obedecer.

Se observaron los desafíos y límites que tiene la población investigada para cuidar y cuidarse y las denominamos "paradójicas" siguiendo a Watzlawick et al. en tanto "la paradoja puede definirse como una contradicción que resulta de una deducción correcta a partir de premisas congruentes"(35). Estas dos premisas son precisamente las prácticas de cuidado que facilitan, paradójicamente, condiciones de riesgo para los adolescentes.

En los relatos de los participantes se encontró el cuidado -en su sentido ontológico de acuerdo con Boff ${ }^{(36)}$ - a partir de expresiones de preocupación e interés por un semejante. Los varones adolescentes mostraron apertura para el cuidado del sufrimiento entre pares, aunque sin herramientas socioemocionales para hacer frente al malestar propio o de otro.

\section{CONCLUSIONES}

Los testimonios que compartieron los entrevistados recuperan experiencias propias y ajenas de sufrimiento psíquico y las prácticas de cuidado entre pares en un ámbito rural mexicano. Se observan tensiones en las representaciones culturales sobre lo que debe ser un hombre y una mujer joven en ese contexto.

Como se pudo apreciar, la relación con sus madres y/o padres son fuentes de sufrimiento psíquico especialmente por la distancia afectiva y las barreras en la comunicación que encuentran con ellos y el sentimiento de abandono que les produce la salida de sus madres al espacio laboral externo a la comunidad.

La incomprensión o falta de aceptación sobre los cambios de rol de las mujeres en la comunidad y, en particular, cómo esto afecta sus relaciones de noviazgo es una más de las fuentes de sufrimiento psíquico.

Los entrevistados tienen dificultades para diferenciar emociones tales como el enojo y la tristeza, así como para el manejo y expresión de estas; esto los lleva a recurrir al consumo de alcohol como medio para sobrellevarlo o bien para abrirse al diálogo con sus pares.

El cuidado entre pares es paradójico, pues sus prácticas de cuidado derivan frecuentemente en situaciones de riesgo tales como pelear o embriagarse. Esta situación se vincula, por un lado, con la dificultad para establecer, de manera espontánea, espacios de confianza entre pares varones $y$, por el otro, por la mayor empatía, escucha o interés que le atribuyen a sus pares mujeres.

Se identificaron cambios en la dinámica rural a partir de la salida al campo laboral de las mujeres y de los adolescentes varones, la dependencia económica al municipio aledaño, así como el mayor nivel educativo de los participantes. Todo lo anterior pone en escena condiciones para el menosprecio o invisibilización de los orígenes indígenas de la localidad, así como condiciones precarias en los habitantes de San Antonio de la Cal que transforman el tejido de la comunidad. 
Todo esto hace evidente la necesidad de construir intervenciones que permitan a los adolescentes varones construir formas de vincularse entre sí a partir de lógicas de cuidado centradas en la afectividad, las cuales fortalezcan el tejido comunitario y apuesten a la elaboración del sufrimiento psíquico y al desarrollo integral de los adolescentes. Así, pensar desde salud colectiva a la luz de los resultados de esta investigación, implica dar cuenta de elementos estructurales que afectan la vida comunitaria y, por lo tanto, la subjetividad. Desde una perspectiva interseccional se reconoce a estos sujetos en tanto hombres, adolescentes, en un contexto rural como depositarios de la desigualdad estructural patriarcal, pero también como posibles agentes de transformación social a partir del cuidado entre pares.

\section{AGRADECIMIENTOS}

Agradecemos el apoyo obtenido para la realización de este proyecto por parte del Consejo Nacional de Ciencia y Tecnología (CONACYT), México, a través del programa Nacional de Posgrados de Calidad, por medio de la Beca de Maestría que obtuvo el Maestro en Educación para la Ciudadanía, Hernando Hernández Nava, para la realización de sus estudios y de la tesis que dio lugar a este artículo. Asimismo, queremos agradecer el apoyo financiero obtenido por el Fondo de Proyectos Especiales de Rectoría, de la Universidad Autónoma de Querétaro, México.

\section{REFERENCIAS BIBLIOGRÁFICAS}

1. Hernández MTDJ, De la Torre J, Espinoza M, Lara B, Gutiérrez S. El concepto de Salud Mental desde el Estado mexicano. Revista Educación y Desarrollo. 2016;(37):89-93.

2. Organización Mundial de la Salud. Salud mental. [Internet]. 2013 [citado 20 mar 2019]. Disponible en: https://tinyurl.com/v8bdskf.

3. Augsburguer AC. La inclusión del sufrimiento psíquico un desafío para la epidemiología. Psicologia \& Sociedade. 2004;16(2):71-80.

4. Galende E. Psicofármacos y salud mental: La ilusión de no ser. Buenos Aires: Lugar Editorial; 2008.

5. Menéndez EL. Morir de alcohol: saber y hegemonía médica. México: Alianza Editorial Mexicana; 1990.

6. Granda E. ¿Qué llamamos salud colectiva hoy? Revista Cubana de Salud Pública. 2004;30(2):148159.

7. Cortés D, Parra G. La ética del cuidado: Hacia la construcción de nuevas ciudadanías. Psicología desde el Caribe. 2009,23:183-213.
8. Hernández-Castañeda M. Varones con o sin empleo la construcción de las masculinidades en Chihuahua. [Tesis de doctorado]. Tlaquepaque, Jalisco: ITESO; 2013.

9. Vale de Almeida M. The hegemonic male. Masculinity in a Portuguese Town. Oxford: Berghahn Books, 1996:4-5.

10. Organización Panamericana de la Salud. Editorial: La importancia de abordar la masculinidad y la salud de los hombres para avanzar hacia la salud universal [Internet]. 2019 [citado 29 mar 2020] Disponible en: https://tinyurl.com/vx2d6dt.

11. Secretaría de Salud. Perfil epidemiológico de la población adolescente en México 2010. México: Secretaría de Salud; 2011.

12. Figueroa JG, Flores N. Prácticas de cuidado y modelos emergentes en las relaciones de género: La experiencia de algunos varones mexicanos. La ventana. 2012;4(35):7-57.

13. Jarquin NH, Castellanos JA, Sangerman-Jarquín DM. Pluriactividad y agricultura familiar: retos del desarrollo rural en México. Revista Mexicana de Ciencias Agrícolas. 2017;8(4):949-963.

14. Zamudio FJ, Corona A, López ID. Un índice de ruralidad para México. Espiral. 2008;14(42):179214

15. Salazar A. Vivir entre la Peña y la marginación. El Universal [Internet]. 9 nov 2017 [citado 9 nov 2017]. Disponible en: https://tinyurl.com/t8nxptc.

16. Instituto Nacional de Estadística y Geografía. Censo de Población y Vivienda 2010 [Internet]. México: INEGI; 2010 [citado 9 jun 2016] Disponible en: https://tinyurl.com/us5poht.

17. Hernández-Nava H. Prácticas de cuidado de la salud mental entre pares como un componente necesario para la construcción de ciudadanía activa de los hombres adolescentes: Un estudio exploratorio-descriptivo. [Tesis de Maestría]. Querétaro: Universidad Autónoma de Querétaro; 2019. 
18. Hernández-Nava H, Garbus P. Prácticas de cuidado de la salud mental entre pares como componente necesario para la construcción de ciudadanía activa de los hombres adolescentes: análisis y propuesta de intervención. En: Diez-Martínez E, (comp.). Aproximaciones y perspectivas actuales en educación para la ciudadanía: aportes desde la intervención y la investigación. México: Eólica Grupo Editorial/Universidad Autónoma de Querétaro; 2019. p. 95-117.

19. Hernández-Nava $H$, Garbus $P$. Prácticas de cuidado de la salud mental entre pares en hombres adolescentes de San Antonio de la Cal, Qro, Mex. IX Congreso Internacional de Investigación y Práctica Profesional en Psicología, XXIV Jornadas de Investigación de la Facultad de Psicología, XIII Encuentro de Investigadores en Psicología del MERCOSUR; diciembre 2017. Buenos Aires: Facultad de Psicología, Universidad de Buenos Aires.

20. Rivas HE. ¿El varón como factor de riesgo? Masculinidad y mortalidad por accidentes y otras causas violentas en la Sierra de Sonora. Estudios Sociales: Revista de Alimentación Contemporánea y Desarrollo Regional. 2005;13(26):28-65.

21. Núñez G. Masculinidad, ruralidad y hegemonías regionales: reflexiones desde el norte de México. Región y Sociedad. 2017;29(5):75-113.

22. López GL, Marzolla ME, Olivarez MS. Los espacios de construcción de subjetividad en la escuela media. V Congreso Internacional de Investigación y Práctica Profesional en Psicología, XX Jornadas de Investigación, IX Encuentro de Investigadores en Psicología del MERCOSUR. Buenos Aires: Facultad de Psicología, Universidad de Buenos Aires; 2013.

23. Burin M, Meler I. Varones: género y subjetividad masculina. Buenos Aires: Paidós; 2000.

24. Castaño JA. Masculinidades y sexualidades de corteros de caña en el municipio de CandelariaValle. Sociedad y Economía. 2011;(21):221-241.

25. Viveros M. De quebradores y cumplidores: Sobre hombres, masculinidades y relaciones de género en Colombia. Colombia: Universidad Nacional de Colombia; 2002.

26. Cevallos JP. Intersecciones de género, clase, etnia y raza: Un diálogo con Mara Viveros. Iconos: Revista de Ciencias Sociales. 2017;(57):117-121.

27. Orcasita L, Uribe AF. La importancia del apoyo social en el bienestar de los adolescentes. Psychologia Avances de la Disciplina. 2017;4(2):69-82.

28. Rodríguez E, Megías I, Sánchez E. Jóvenes y relaciones grupales. Madrid: INJUVE, Ancares; 2002.

29. De Keijzer B. El varón como factor de riesgo: masculinidad, salud mental y salud reproductiva. En: Tuñón E. Género y salud en el Sureste de México. México DF: El Colegio de la Frontera Sur, Universidad Juárez Autónoma de Tabasco; 1997. p. 1-15.

30. Lomas C. Los chicos también Iloran: Identidades masculinas, igualdad entre los sexos y coeducación. 3a ed. Barcelona: Ediciones Paidós Ibérica; 2011.

31. Blanco J. Hombres: La masculinidad como factor de riesgo: Una etnografía de la invisibilidad. [Tesis de Doctorado]. Sevilla: Universidad Pablo de Olavide; 2012.

32. Heilman B, Barker G, Harrison A. La caja de la masculinidad: Un estudio sobre lo que significa ser hombre joven en Estados Unidos, el Reino Unido y México. Washington DC: Promundo US, Uniliver; 2017.

33. Monsiváis C. ¿Pero hubo alguna vez once mil machos? FEM. 1981;(18):9-20.

34. Way N. Deep secrets: boys, friendships, and the crisis of connection. Cambridge: Harvard University Press; 2011.

35. Watzlawick P, Beavin J, Jackson DD. Teoría de la comunicación humana. Barcelona: Herder; 1991.

36. Boff L. El cuidado esencial. Madrid: Trotta; 2002. 